\title{
Maturation of the gastric microvasculature in Xenopus laevis (Lissamphibia, Anura) occurs at the transition from the herbivorous to the carnivorous lifestyle, predominantly by intussuceptive microvascular growth (IMG): a scanning electron microscope study of microvascular corrosion casts and correlative light microscopy
}

\author{
Alois Lametschwandtner • Monika Höll • \\ Heidi Bartel • Vipavee Anupunpisit • \\ Bernd Minnich
}

Received: 21 July 2011/Accepted: 27 November 2011/Published online: 12 January 2012

(C) The Author(s) 2012. This article is published with open access at Springerlink.com

\begin{abstract}
The microvascular bed of the stomach of Xenopus laevis and the changes it undergoes when the herbivorous tadpole becomes a carnivorous adult were studied by scanning electron microscopy of vascular corrosion casts and light microscopy of stained tissue sections. In tadpoles an upper and a lower gastric artery supplied, and upper, middle and lower medial and lateral gastric veins drained the vertically extending stomach. During metamorphosis, the stomach gained a horizontal craniocaudal extension and vessels accordingly become dorsal and ventral gastric arteries, and anterior, middle and posterior gastric veins, respectively. Up to stage 64 (late climax) mucosal capillaries formed a polygonal network of wide immature-looking capillaries ensheathing gastric glands in a basket-like manner. From stage 64 onwards, blood vessels of the stomach appeared mature, revealed a clear hierarchy and were correlated closely with the histomorphology of the stomach, which had also gained the adult pattern. Within the gastric mucosa, ascending arterioles branched in a fountain-like pattern into wide subepithelial capillaries establishing a centripetal blood flow
\end{abstract}

\footnotetext{
A. Lametschwandtner $(\square) \cdot$ M. Höll · H. Bartel · B. Minnich Vascular and Muscle Research Unit, Division of Zoology and Functional Anatomy, Department of Organismic Biology, University of Salzburg, Hellbrunnerstrasse 34, 5020 Salzburg, Austria

e-mail: Alois.Lametschwandtner@sbg.ac.at

V. Anupunpisit

Department of Anatomy, Faculty of Medicine,

Shrinakharinwirot University, 114 Sukhumvit 23,

Bangkok, Thailand
}

along the gastric glands, which makes an ultrashort control loop of glandular cells within the branched tubular gastric glands very unlikely. Formation of the stomach external muscular layer started at stage 57 when smooth muscle cells locally formed a single longitudinal and one-to-two single circular layers. Abundant signs of intussusceptive microvascular growth and rare vascular sprouts in vascular corrosion casts indicated that the larval-to-adult microvascular pattern formation of the stomach of Xenopus laevis Daudin occurs predominantly by non-sprouting angiogenesis.

Keywords Xenopus - Stomach · Microvasculature · Non-sprouting angiogenesis - Vascular casting . Scanning electron microscopy

\section{Introduction}

We have a detailed knowledge of the development of the arterial and venous system of Xenopus laevis as revealed by light microscopy of serially sectioned embryos and larvae (Millard 1945, 1949), and by confocal microscopy of DiIlabelled embryos (Levine et al. 2003). Weisz (1945a, b) described the main vessels in larval Xenopus, and Millard (1941) studied the macrovasculature and its abnormalities and variations in the adult animal (Millard 1942), but neither of these studies focused on the stomach microvascularization and the changes it undergoes when the suspension feeding herbivorous larva (tadpole) metamorphosizes and becomes a carnivorous postmetamorphic animal. 
Here, we analyze vascular corrosion casts of the larval stomach (manicotto glandulare; Lambertini 1929; Ueck 1967) of larval stages 55-57 (premetamorphosis), i.e., before onset of metamorphosis associated histolyis in the stomach; of stages 62-64 (metamorphic climax), i.e., just before the stomach epithelium is differentiated and the pyloric glands start secretion; and of adult Xenopus laevis using the excellent resolution and high depth of focus of the scanning electron microscope (Murakami 1971; Lametschwandtner et al. 1990; Aharinejad and Lametschwandtner 1992; Motta et al. 1992). Additionally, we analyze stained sections of paraplast-embedded gastric tissue and attribute cast blood vessels to defined tissue layers of the stomach.

The study aimed to (1) demonstrate microvascular pattern formation from the larval to the adult stomach in the model organism Xenopus laevis, and (2) address the question of which mode(s) of angiogenesis, i.e., sprouting and/or nonsprouting angiogenesis (=intusssceptive microvascular growth, IMG) and its facets intussusceptive arborization (IA), intussusceptive branch remodeling (IBR), and intussusceptive pruning (IPR) (Patan et al. 1992; Djonov and Makanya 2004; Burri et al. 2004; Makanya et al. 2009) drive these processes.

\section{Materials and methods}

Animals

Tadpoles of developmental stages 55-57 (body weight 190-1010 mg) and stages 62-64 (body weight 110-640 mg) and adults (two males, five females, body weight 46-126 g) of the South African Clawed Toad, Xenopus laevis Daudin were studied. Tadpoles were reared in aerated tap water at $23^{\circ} \mathrm{C}$ under artificial light (L:D 12:12) and fed nettle powder every other day. Tadpole staging was according to Nieuwkopp and Faber (1967).

\section{Histomorphology}

\section{Tadpoles}

Tadpoles were killed by an overdose of tricaine methanosulfate (MS222, 0.01\%; Sigma , St. Louis, MO). After recording body weight (BW, mg), snout-vent-length (SVL, $\mathrm{mm}$ ) and total length (TL, mm), tadpoles were fixed in supine position on a wax-plate, the skin overlying the pericard and the pericard were cut open with microscissors, and a glass cannula was introduced by the use of a micromanipulator via a cut in the apex of the ventricle into the arterial trunk. The cannula was fixed by a ligature of surgical suture material (10-0; Ethicon, Neuchatel,
Switzerland) and both atria were cut open to allow for drainage of blood. The vascular system was then rinsed with Amphibian Ringer solution (Adam and Czihak 1964) at room temperature $\left(20^{\circ} \mathrm{C}\right)$ via the glass cannula using a syringe pump (flow rate $3-7 \mathrm{ml} / \mathrm{h}$ ) until clear reflux escaped from the opened venous sinus. Subsequently, specimens were fixed by perfusion with Bouin's solution, dehydrated in a graded series of ethanol and embedded in paraplast. Transverse and longitudinal serial sections (7 $\mu \mathrm{m}$ thick) of whole tadpoles were stained according to Masson-Goldner or Cleveland-Wolfe (Böck 1989). Stained tissue sections were examined with a light microscope (Olympus BX 51) and images were taken with a digital camera (Color View III; Soft Imaging Systems, Münster, Germany). Brightness and contrast of recorded images were adjusted using Adobe Photoshop 7.0.

\section{Adults}

Animals killed by immersion in an overdose of an aqueous solution of tricaine methansulfonate (MS 222; 0.1\%; Sigma) were fixed by perfusion with Bouin's solution via the arterial trunk. The stomach with part of esophagus and duodenum was then excised, postfixed in fresh fixative, dehydrated in a graded series (50-100\%) of ethanol, and embedded in paraplast. Thick transverse and longitudinal sections (7-10 $\mu \mathrm{m}$ thick) were stained with Goldner's trichrome stain (for details see Adam and Czihak 1964).

Vascular casting

\section{Tadpoles}

For preparatory steps preceding resin injection see section on Histomorphology. After clear reflux escaped from the opened atria Mercox CL-2B (Ladd Research, Burlington, VT), diluted with monomeric methylmethacrylate $(4+1$, $\mathrm{v} / \mathrm{v}, \quad 10 \mathrm{ml}$ monomeric methylmethacrylate contained $0.85 \mathrm{~g}$ initiator paste MA) was injected with an syringe pump $(5-7 \mathrm{ml} / \mathrm{h})$ until the effluent resin became highly viscous. Animals were left for at least $30 \mathrm{~min}$ at room temperature $\left(20^{\circ} \mathrm{C}\right)$ to allow polymerization of the injected resin, then placed in a water bath $\left(60^{\circ} \mathrm{C}, 12 \mathrm{~h}\right)$ for tempering, and finally macerated in $\mathrm{KOH}(7.5 \%, 12-24 \mathrm{~h}$, $20^{\circ} \mathrm{C}$ ). When maceration was completed, vascular casts were transferred via several passages of distilled water into formic acid $\left(5 \%, 5-10 \mathrm{~min}, 20^{\circ} \mathrm{C}\right)$, rinsed again in several passages of distilled water, frozen in distilled water and freeze-dried in a Lyovac GT2 (Leybold Heraeus, Cologne, Germany). Dry specimens were mounted onto stubs using the "conductive bridge method" (Lametschwandtner et al. 1980) evaporated with carbon and gold, and finally sputtered with a thin layer of gold. Coated specimens were 
investigated with a scanning electron microscope (Stereoscan 250, Cambridge Scientific Instruments, Cambridge, UK and/or XL-30, FEI, Eindhoven, The Netherlands) at an accelerating voltage of $10 \mathrm{kV}$.

After initial documentation and analysis of the superficial (external) vascular layers of the stomach, specimens were removed from specimen stubs, submerged in an appropriately sized plastic container filled with bidistilled water, orientated properly, and frozen at $-20^{\circ} \mathrm{C}$. Iceembedded specimen then were frozen onto wooden specimen holders and sectioned either transversely, longitudinally, tangentially or horizontally with a mini-wheel-saw
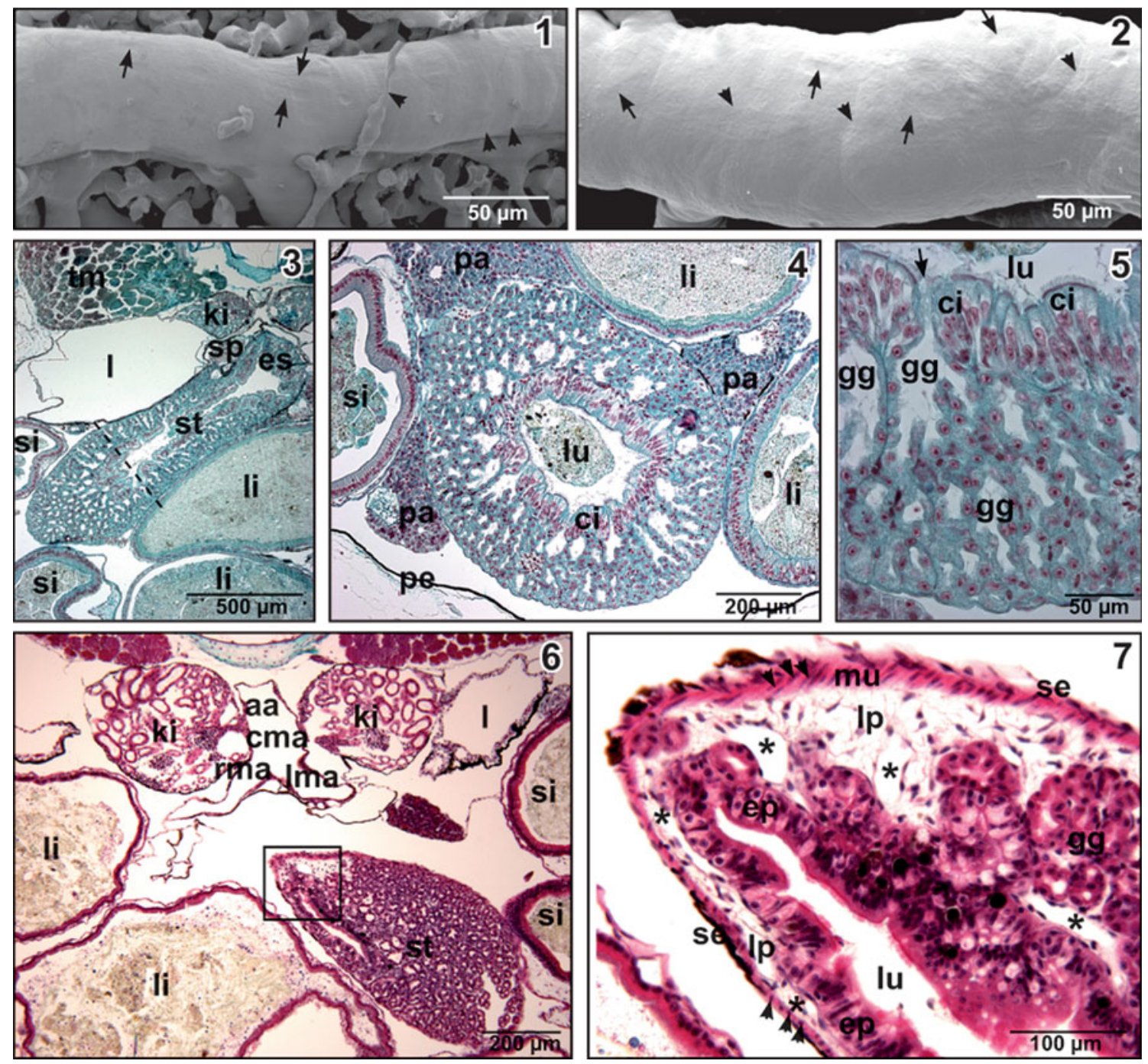

Figs. 1-7 Fig. 1 Scanning electron micrograph (SEM) of the surface of a resin cast of an artery displaying characteristic longish endothelial cell nuclei imprints (arrows) orientated parallel to the long axis of the vessel. Note the slight circular narrowings caused by the circularly arranged vascular smooth muscle cells (arrowheads). Fig. 2 SEM of the surface of a resin cast of a vein displaying characteristic oval-to-roundish endothelial cell nuclei imprints (arrows) orientated randomly. Note faint circularly orientated imprints (arrowheads). Fig. 3 Histomorphology of the stomach of larval Xenopus at Nieuwkopp and Faber (NF) stage 55. Transverse section $(7 \mu \mathrm{m})$ through whole tadpole. Cleveland-Wolfe-staining. Note the transition from the esophagus (es) to the glandular stomach (Manicotto glandulare) ( $s t$ ). ki Kidney, li large intestine, $l$ lung, $s i$ small intestine, $s p$ spleen, $t m$ trunk muscles. Fig. 4 Stomach at NF stage 55. Transverse section at the level indicated in Fig. 3 (dashed line). Note the ciliated ( $c i$ ) gastric epithelium bordering the lumen

(lu). li Large intestine, pa pancreas, pe pigmented peritoneum, si small intestine. Fig. 5 Wall of the stomach at NF stage 55. Note a branched tubular gastric gland ( $g g$ ) opening (arrow) into the lumen (lu). ci Ciliated columnar gastric epithelium. Fig. 6 Histomorphology of the stomach of larval Xenopus at Nieuwkopp and Faber (NF) stage 55. Transverse section $(7 \mu \mathrm{m})$ through whole tadpole at the level of the origin of the celiac-mesenteric artery $(\mathrm{cma})$ from the abdominal aorta $(a a)$. Goldner staining. Note origin of left (Ima) and right mesenteric artery ( $r m a)$ from the cma. ki Kidney, li large intestine, $l$ lung, si small intestine, st stomach. Fig. 7 Tangential section through the proximal portion of the stomach. Detail from Fig. 6 (boxed area) showing the single circular layer of smooth muscle cells forming the muscularis. Note the tangentially and transversely sectioned smooth muscle cells (arrowheads). Asterisks Gastric vessels, $e p$ gastric epithelium, $g g$ gastric gland, $l p$ lamina propria, lu lumen, mu muscularis, se serosa 
placed in the cryo-chamber of a cryo-microtome (Lametschwandtner and Lametschwandtner 1992). Sectioned iceembedded specimens were allowed to thaw in distilled water, and were then cleaned, and refrozen in bidistilled
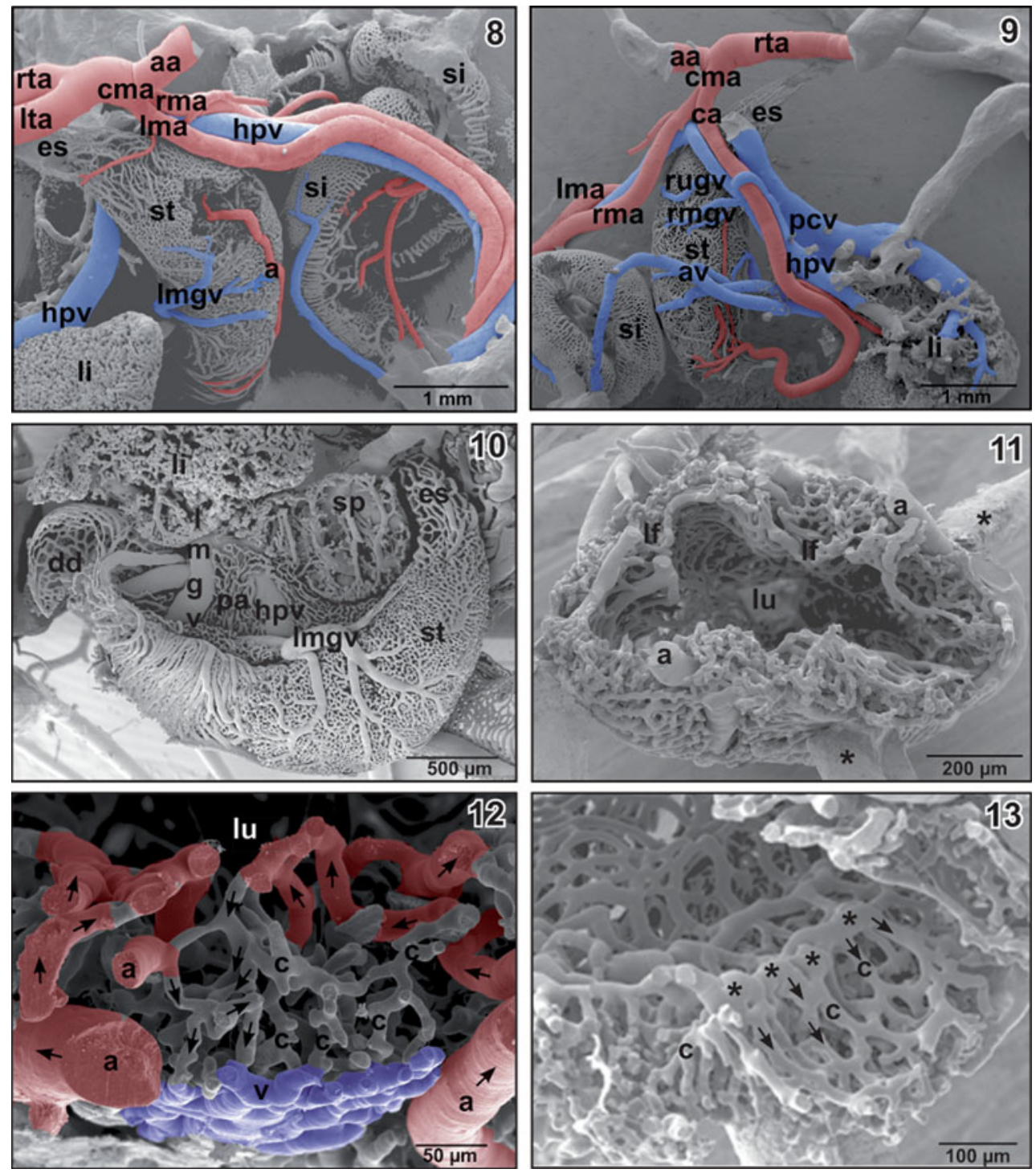

Figs. 8-13 Fig. 8 SEM of gross arterial supply and venous drainage of the larval stomach at NF stage 56. Lateral view. Cranial is to the left. Part of liver, pancreas and intestines are removed to expose the lateral (left) surface of the dorso-ventral orientated stomach. Note the large left middle gastric vein (lmgv) and the artery (a) running close along the posterior curvature of the slightly sickle-shaped stomach. aa Abdominal aorta, cma celiac-mesenteric artery, es esophagus, $h p v$ hepatic portal vein, li liver, lma left mesenteric artery, lta left thoracic aorta, rta right thoracic aorta, si small intestine, st stomach. Fig. 9 Same as Fig. 6, but lateral view at the medial (right) surface of the stomach. Cranial is to the right. Kidneys with most of abdominal aorta, posterior caval vein, intestines, pancreas and liver were removed to expose origin, course and branches of the celiac artery ( $c a$ ). a a Abdominal aorta, av abdominal vein, cma celiacmesenteric artery, es esophagus, $h p v$ hepatic portal vein, lma left mesenteric artery, li liver, $p c v$ posterior caval vein, rma right capillaries (c). Arrows indicate direction of blood flow water for subsequent freeze-drying. Further steps in specimen preparation were as described above. As required, specimens were sectioned repeatedly to expose interesting vascular territories.

mesenteric artery, rugv right upper gastric veins, rmgv right middle gastric vein, si small intestine, st stomach. Fig. 10 Vascular corrosion cast (VCC) of the microangioarchitecture of the larval stomach at NF stage 55. Lateral aspect. Cranial is at top, dorsal is at right. Note the large calibre of the left middle gastric vein $(l m g v)$. $d d$ Duodenum, es esophagus, $l i$ liver, $p a$ pancreas, $h p v$ hepatic portal vein, $s p$ spleen, $s t$ stomach. Fig. 11 VCC of microvascular pattern of the larval stomach at NF stage 55. Transverse section at the level of the corpus. Note the longitudinal folds $(l f)$ protruding into the lumen $(l u)$. a Artery. Asterisks "Conductive bridges". Fig. 12 SEM of microvascular anatomy of the stomach wall at NF stage 55. Arterial vessels are colored red, venous vessels are colored blue. Arrows mark direction of blood flow. a Artery, $c$ capillary, $l u$ lumen, $v$ vein. Fig. 13 Subepithelial capillary bed at NF stage 55. Luminal view. Note the wide vessels (asterisks; cf. Fig. 7) giving off centrifugally running 

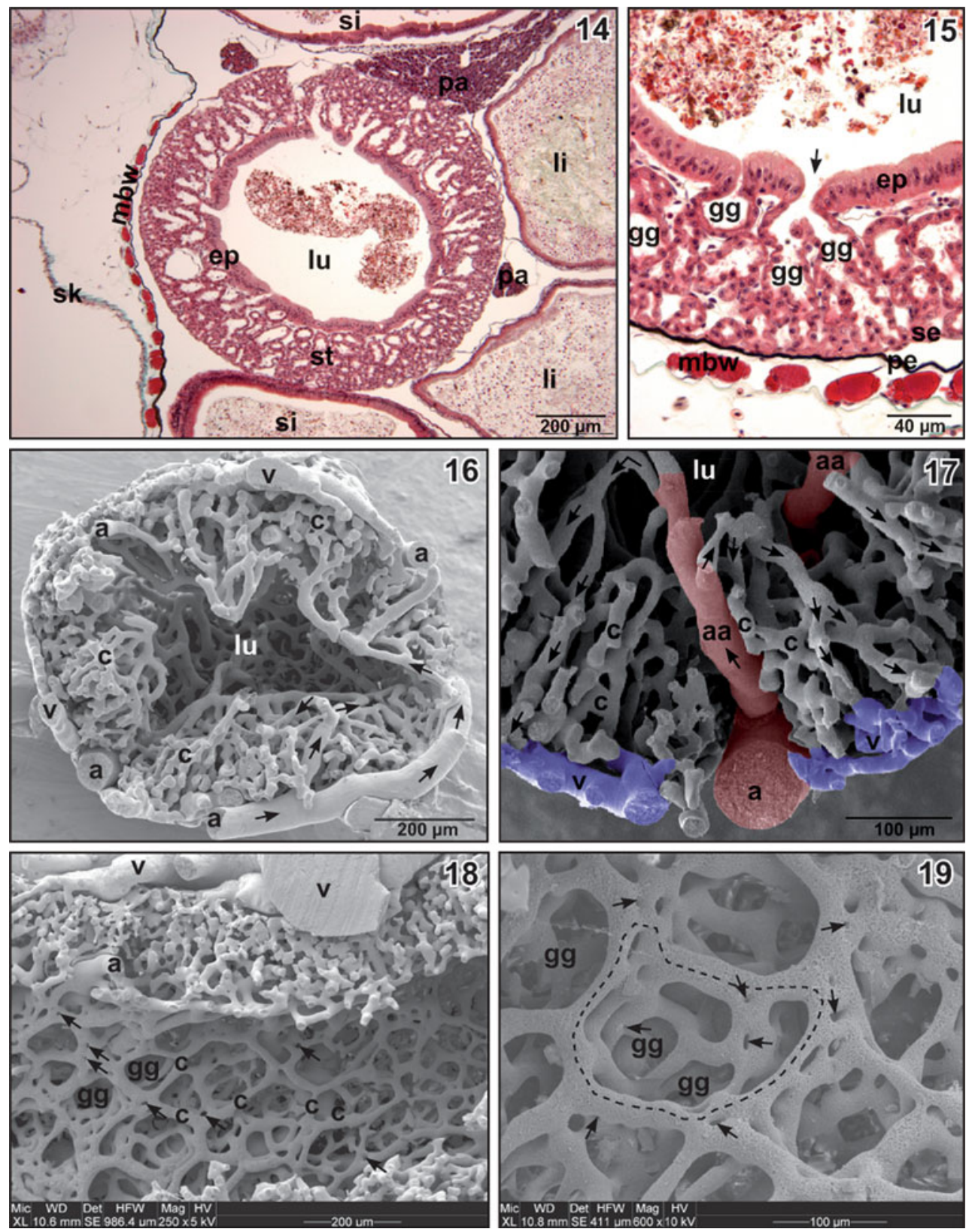

Figs. 14-19 Fig. 14 Histomorphology (Goldner staining) of the larval stomach at NF stage 57 . Transverse section $(7 \mu \mathrm{m})$. Note the thick gastric mucosa composed mainly of wide tubular glands. $e p$ Columnar mucosal epithelium, li large intestine, lu lumen, $m b w$ muscles of the body wall, pa pancreas, si small intestine, sk skin, $s t$ stomach. Fig. 15 Detail from Fig. 8. Note wide branched tubular gastric glands $(g g)$ opening (arrow) into the lumen $(l u)$ of the stomach. ep Columnar mucosal epithelium, $m b w$ muscles of the body wall, pe pigmented peritoneum, se serosa. Fig. 16 Microangioarchitecture of the stomach at NF stage 57. Transverse section at the level of the corpus. Note the wide immature imposing capillaries (c) embracing tubular gastric glands. $a$ Artery, $l u$ lumen, $v$ vein. Arrows indicate direction of blood flow. Fig. 17 Arteriolar supply of the glandular mucosa at NF stage 57. Transverse section at the level

of the corpus. Detailed view. Note two arterioles ( $a a)$ running centripetally towards the lumen $(l u)$ where they capillarize into centrifugally running capillaries $(c)$ ensheathing the tubular gastric glands. Arrows indicate direction of blood flow. $a$ Artery, $v$ vein. Fig. 18 Microangioarchitecture of the stomach at NF stage 57. Longitudinal section. Luminal view. The wide meshes of the subepithelial capillary bed $(c)$ indicate the locations of tubular gastric glands $(g g)$. Note the many small holes (arrows) in the wide capillaries reflecting ongoing intussuceptive microvascular growth (IMG). $a$ Artery, $v$ vein. Fig. 19 Detail from Fig. 18. Vascular pattern of the subepithelial capillary bed of the gastric mucosa at NF stage 57 . Luminal view. Dashed line outlines the capillaries ensheathing a branched tubular gastric gland $(g g)$. Note the many signs of ongoing IMG (arrows) 

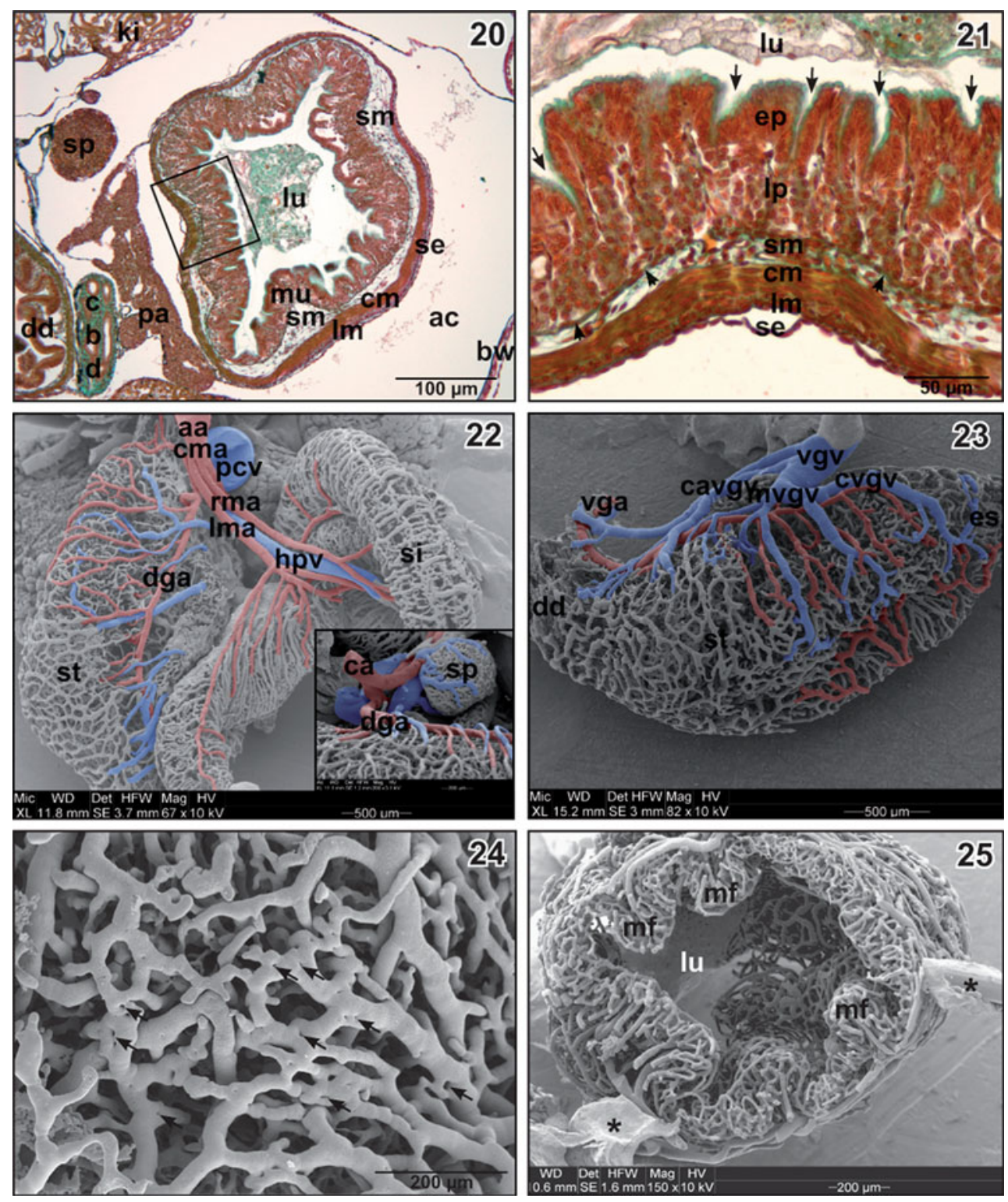

Figs. 20-25 Fig. 20 Histomorphology (Goldner stain) of the stomach at NF stage 64 . Transverse section $(7 \mu \mathrm{m})$. Note the presence of a submucosa $(\mathrm{sm})$ with loose connective tissue. ac Abdominal cavity, $b w$ body wall, $c b d$ common bile duct, $\mathrm{cm}$ circular layer of muscularis, $d d$ duodenum, $k i$ kidney, $l m$ longitudinal layer of muscularis, $l u$ lumen, $m и$ mucosa, $p a$ pancreas, se serosa, $s m$ submucosa, $s p$ spleen. Fig. 21 Detail of Fig. 20 (boxed area). Note gastric pits (arrows) and thin muscular layer of the mucosa (arrowheads). $\mathrm{cm}$ Circular layer of muscularis, ep epithelium, $l m$ longitudinal layer of muscularis, $l p$ lamina propria, lu lumen, se serosa, sm submucosa. Fig. 22 SEM/ VCC of gross arterial supply and venous drainage of the larval stomach at NF stage 64. Dorsal view. Cranial is on top. Part of liver, pancreas, intestines and kidneys are removed to expose the dorsal surface of the horizontally extending stomach. Note the dorsal gastric artery $(d g a)$ running close along the inner curvature of the slightly sickle-shaped stomach. Inset Origin of the dorsal gastric artery (dga) from the celiac artery ( $c a)$. a a Abdominal aorta, cma celiacmesenteric artery, $h p v$ hepatic portal vein, $l i$ large intestine, lma left mesenteric artery, $p c v$ posterior caval vein, rma right mesenteric artery, $s i$ small intestine, $s p$ spleen, $s t$ stomach. Fig. 23 Microvascular patterns of the isolated larval stomach at NF stage 64. Ventral view. Cranial is to the right, medial is on top. Note the course of the ventral gastric artery ( $v g a)$ ascending from the duodenum to the stomach. Cranial (cvgv), middle (mvgv) and caudal ventral gastric veins (cavgv) form a strong ventral gastric vein $(v g v)$. $d d$ Duodenum, es esophagus, $s t$ stomach. Fig. 24 Lateral region of the stomach at NF stage 64 still displaying many signs of ongoing intussusceptive microvascular growth (arrows). Fig. 25 Microangioarchitecture of the stomach at NF stage 64 (late metamorphic climax). Transverse section at the level of the corpus. Note the prominent gastric mucosal folds $(\mathrm{mf})$ bulging into the stomach lumen $(l u)$. Asterisks mark "conductive bridges" 


\section{Adults}

For preparatory steps preceding resin injection, see above. After clear reflux from the opened atria, 5-7 ml Mercox$\mathrm{Cl}-2 \mathrm{~B}$ was injected with manual pressure. All further procedures were the same as described for tadpoles.

For easy identification of cast arteries and veins in the scanning electron micrographs, vessels were colored red (arteries) or blue (veins) using Photoshop 7.0 (Adobe; http://www.adobe.com/). Arteries were identified by their characteristic ovoid-to-longish endothelial cell nuclei imprints (ECNI) that orientate parallel to the vessels' long axis (Fig. 1), while veins were identified by roundish ECNIs that orientate randomly (Fig. 2).

The study was approved by the Ethics Committee of the University of Salzburg.

\section{Results}

The premetamorphic stomach

At stage 55 the stomach wall consisted, from luminal to abluminal, of a simple locally ciliated columnar epithelium, wide branched tubular glands beneath, a very thin lamina propria in between, one layer of circularly arranged smooth muscle cells and a thin serosa composed of squamous epithelial cells forming the interface to the abdominal cavity (Figs. 3-6). The single layer of smooth muscle cells was not yet vascularized and a submucosa was still lacking (Figs. 6, 7).

An upper gastric artery, which branched off the celiac artery just after its origin from the celiac-mesenteric artery and a lower gastric artery, which branched off the desending celiac artery at the level of the pyloric region supplied the dorso-ventrally extending larval stomach (Figs. 8, 9). Upper, middle lower gastric veins drained its right (=lateral) portion into the hepatic portal vein (Fig. 8) and its left (=medial) portion into the abdominal vein (Figs. 9, 10).

Vascular corrosion casts (VCC) of the stomach crosssectioned at midlength revealed longitudinal folds, and a gastric mucosa with varying thickness along its circumference (Fig. 11). A three-dimensional network of wide, immature-looking, capillaries embraced the densely packed branched tubular gastric glands (Figs. 12, 13). In general, vessels within the mucosa appeared rather uniform, and only larger arteries and veins could be differentiated clearly. Mucosal capillaries merged into venules that traversed the lamina propria and finally formed the origins of upper, middle and lower gastric veins (Figs. 8-10).

In stage 57 , the number of ciliated cells of the gastric epithelium decreased (Fig. 14). Branched tubular gastric glands opened into the lumen (Fig. 15). Cast arteries and veins now could be differentiated more easily by their ECNI, but more often by their branching/merging patterns and dimensions. Arteries ran along the serosal side, gave off arterioles that ascended through the lamina propria and capillarized subepithelially in a fountain-like pattern giving rise to centrifugal blood flow patterns (Figs. 16, 17). Subepithelial capillaries formed meshes of different sizes (Figs. 18, 19), and capillaries and venules showed many signs of ongoing IMG (Fig. 19, arrows). Venules descended through the lamina propria and collected blood from the subepithelial capillary bed and the wide capillaries embracing the tubular glands (Figs. 16, 17). The deep portions of the gastric glands drained directly into venules at the base of the lamina propria (Fig. 17).

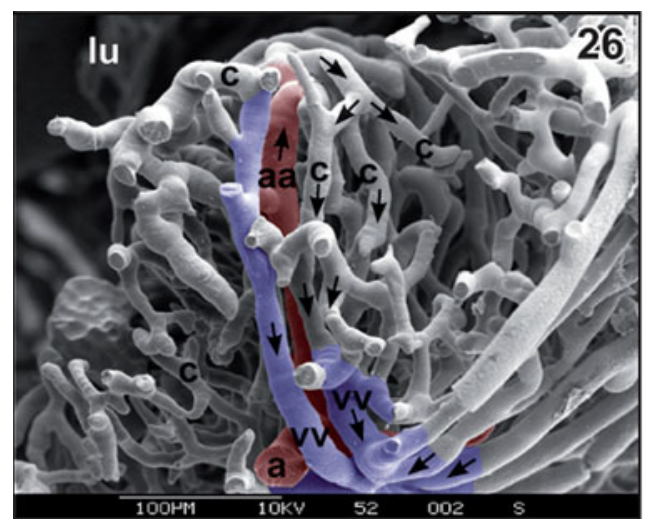

Figs. 26, 27 Fig. 26 Microvascular anatomy of a mucosal fold at NF stage 64 . Transverse section. Note centripetally ascending arteriole $(a a)$ and descending centrifugally running venule $(v v)$ draining subepithelial capillaries and capillaries $(c)$ embracing tubular gastric glands. Arterial vessels are colored red, venous vessels are colored

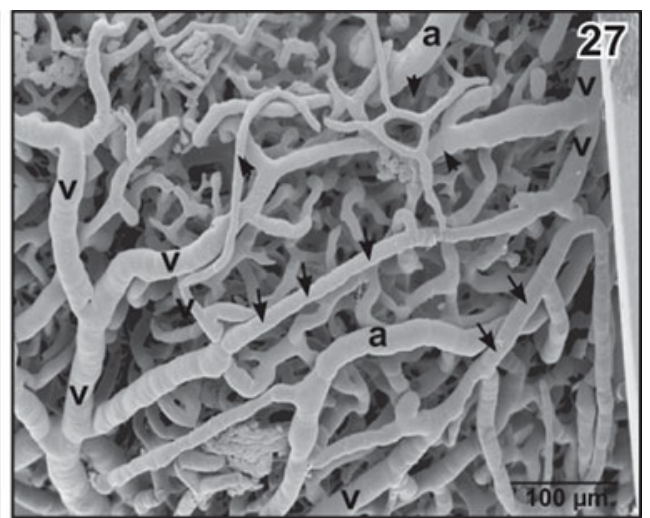

blue. Arrows indicated direction of (centrifugal) blood flow. a Artery, $l u$ lumen. Fig. 27 Microvasculature of the stomach at NF stage 64. Detail. Serosal aspect. Note the few capillaries (arrowheads) of the muscular layer and two veno-venous anastomoses (arrows). a Artery, $v$ vein 

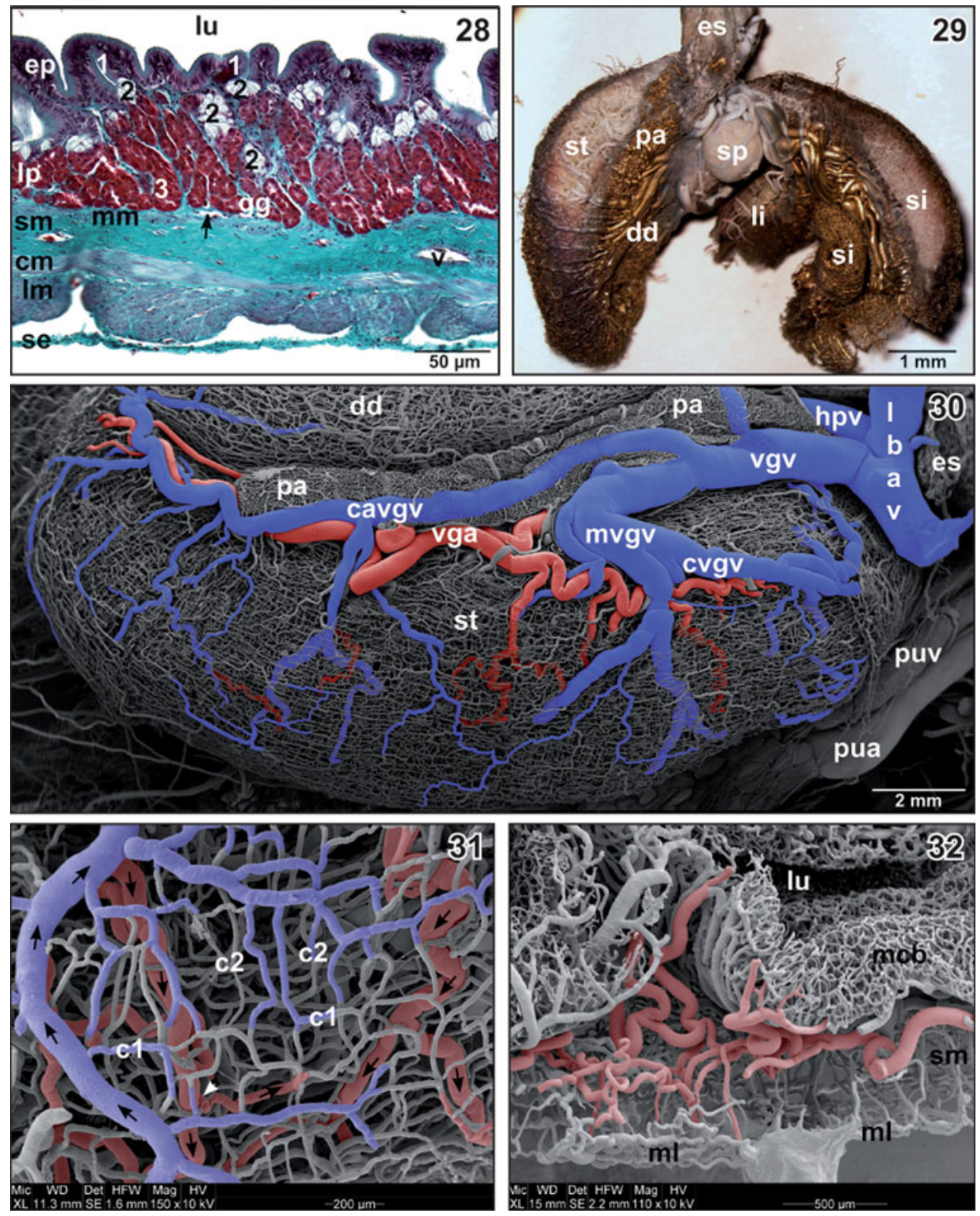

Figs. 28-32 Fig. 28 Histomorphology (Goldner stain) of the wall of the stomach in adult Xenopus. Transverse section $(7 \mu \mathrm{m}) . \mathrm{cm}$ Circular layer of muscularis, ep epithelium, lp lamina propria with gastric glands ( $g g$ ) composed of neck cells ( 1 mucus producing cells), oxyntic cells ( 2 gastric acid producing cells) and chief-cells (3 pepsinogen-producing cells), $l m$ longitudinal layer of muscularis, $l u$ lumen, $\mathrm{mm}$ muscular layer of mucosa, $\mathrm{sm}$ submucosa, se serosa, $v$ vein. Arrow Subepithelial blood vessel. Fig. $29 \mathrm{VCC} /$ stereomicroscopy of the digestive tract in adult Xenopus. Dorsal aspect. $d d$ Duodenum, es esophagus, li large intestine, pa pancreas, si small intestine, $s p$ spleen, $s t$ stomach. Fig. $30 \mathrm{VCC} / \mathrm{SEM}$ of microvasculature of the stomach of adult Xenopus. Ventral aspect. Serosal view. Cranial is at the right, lateral is at the bottom. Arterial vessels are colored red, venous vessels are colored blue. cavgv Caudal ventral gastric vein, $c v g v$ cranial ventral gastric vein, $d d$ duodenum, es esophagus, $h p v$ hepatic portal vein, lbav left branch of abdominal vein, $m v g v$ middle ventral gastric vein, $p a$ pancreas, pua pulmonary artery, $p u v$ pulmonary vein, st stomach, $v g a$ ventral gastric artery, $v g v$ ventral gastric vein. Fig. 31 Vascular bed of the muscular layer of the adult stomach. Serosal view. Note the rectangular capillary meshwork with outer capillaries $(c 1)$ paralleling the longitudinal layer and inner capillaries $(c 2)$ paralleling the circular layer of the muscular layer. Arterioles (colored red) located in the submucosa feed muscular capillaries. Arrowhead Origin of a feeding arteriole. Arrows indicate direction of blood flow. Fig. 32 Microangioarchitecture of the wall of the stomach in adult Xenopus. Longitudinal section. Detail view. Note the mucosal capillary bed $(m c b)$ embracing gastric glands, the convoluted arteries (colored red) within the submucosa ( $\mathrm{sm}$ ), and the capillary bed of the muscular layer $(\mathrm{ml})$. lu Lumen 
The metamorphic stomach

At stage 62, folding of the gastric mucosa had increased and mucosal capillaries embracing gastric glands were thinner and appeared more mature than those in previous stages. At stage 64, the structure of the stomach was already adult-like (Fig. 20). Gastric folds and gastric pits were more numerous and a thin muscular layer separated the lamina propria from the underlying loose connective tissue of the submucosa. The submucosa was thicker at the lateral circumference of the stomach than at its medial side (Fig. 20). Several layers of circularly and longitudinally arranged smooth muscle cells formed the muscular layer (Fig. 21). The thin squamous serosa formed the interface to the abdominal cavity (Fig. 21).

At stage 64, the stomach has changed its orientation and has gained an almost horizontal cranio-caudal extension. The previously upper and lower gastric arteries thus now ran along the inner medio-dorsal (dorsal gastric artery, Fig. 22) and the medio-ventral (ventral gastric artery,
Fig. 23) surface of the stomach. The vasculature appeared mature locally, with distinct arteries branching off the gastric arteries and draining veins running circumferentially (Figs. 22, 23), while in other regions abundant signs of ongoing IMG were present (Fig. 24, arrows). The mucosal capillaries formed a meshwork that clearly outlined the mucosal folds and highlighted them (Fig. 25). A close-up of a transverse sectioned gastric fold revealed ascending arterioles that capillarized at the fold crest and descending venules that drained capillaries embracing gastric glands and finally merged with venules at the submucosa (Fig. 26). Locally, a two-dimensional widemeshed network of circularly and longitudinally arranged capillaries was seen paralleling the smooth muscle cell layers (Fig. 27, arrowheads).

\section{The adult stomach}

In adult Xenopus the mucosa consisted of a glandular epithelium with columnar epithelial cells and tubular
Figs. 33-35 Fig. 33

Microangioarchitecture of the stomach in adult Xenopus. Transverse section at the level indicated in Figs. 34 and 35. Overview. Note the intense folding of the gastric mucosa. a a Arteriole, lp lamina propria, $l u$ lumen, $m l$ muscular layer, $s m$ submucosa, $v$ vein. Fig. 34 Vascular corrosion cast of stomach (st) and duodenum $(d d)$. Dorsal aspect. Serosal view. Cranial is to the left. Dashed line indicates level of the transverse section shown in Fig. 33. pa Pancreas, dga dorsal gastric artery, $d g v$ dorsal gastric vein. Fig. 35 Same as Fig. 34, but ventral aspect. $d d$ Duodenum, dga dorsal gastric artery, gda gastro-duodenal artery, $p a$ pancreas, st stomach, $v g a$ ventral gastric artery, $v g v$ ventral gastric vein

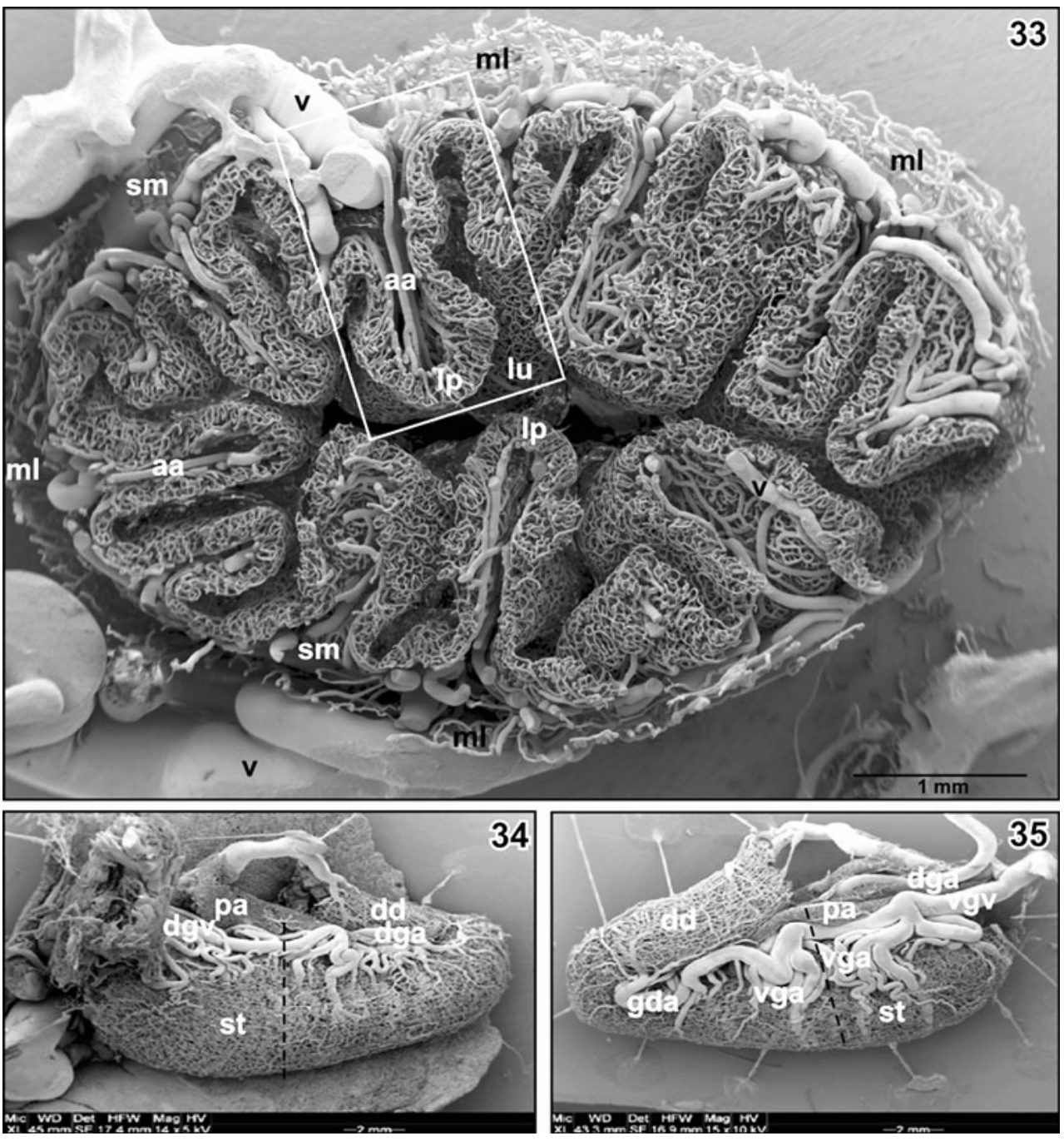


glands composed, from luminal to abluminal, of mucous neck cells, prominent oxyntic cells and pepsinogen-producing chief cells. The lamina propria was bordered by a thin muscularis mucosae composed of an inner circular layer and an outer longitudinal layer of smooth muscle cells separated the lamina propria from the wide submucosa, which was followed by the muscular lamina consisting of an inner circular and an outer longitudinal layer of smooth muscle cells. The squamous serosa formed the interface to the abdominal cavity (Fig. 28).

Dorsal and ventral gastric arteries approached the slightly sickle-shaped stomach from the medial aspect. Anterior, medial and posterior gastric veins drained (Figs. 29, 30). Gastric veins caliber was much larger than that of gastric arteries (Fig. 30). The main branches of gastric arteries and veins located in the submucosa and ran

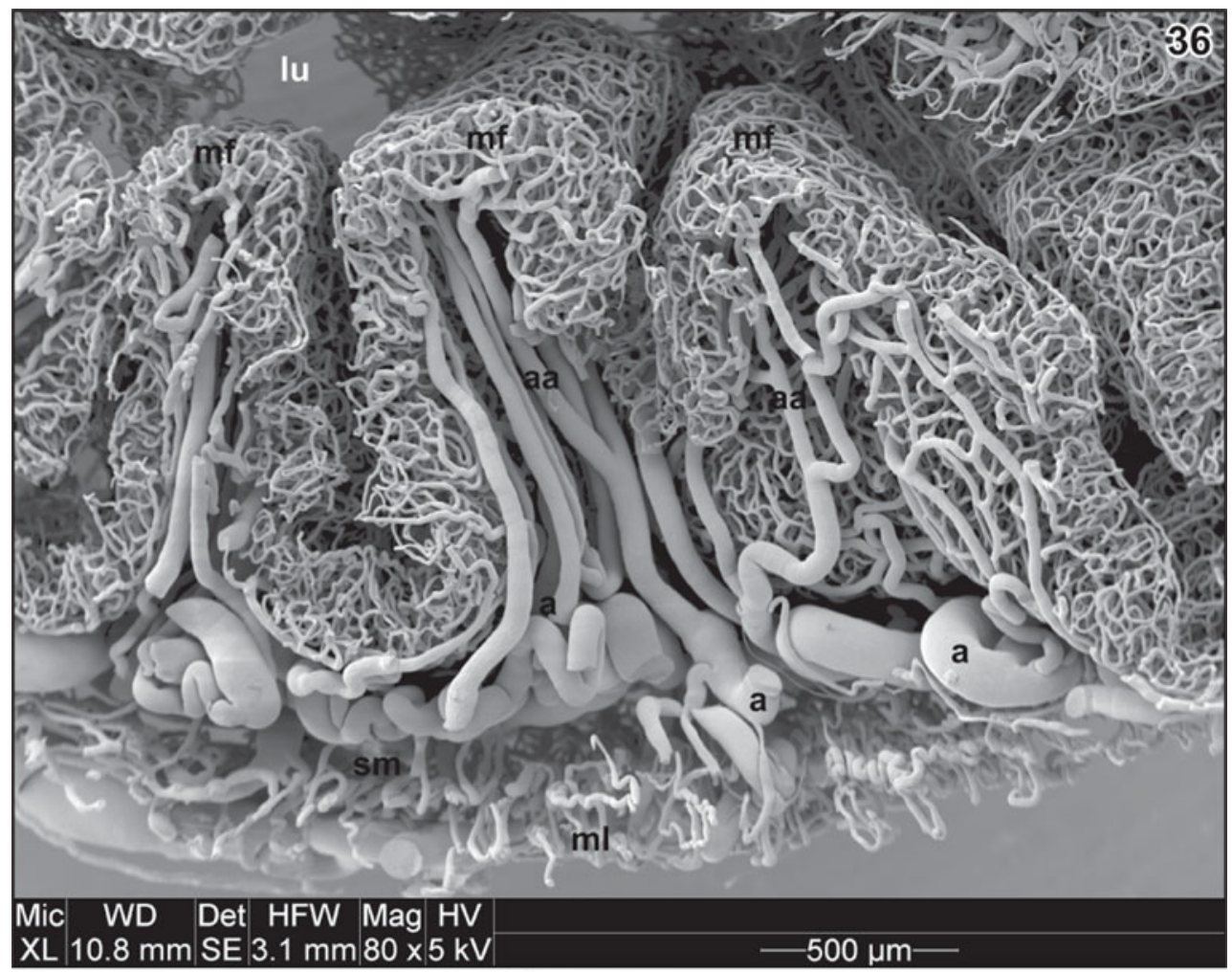

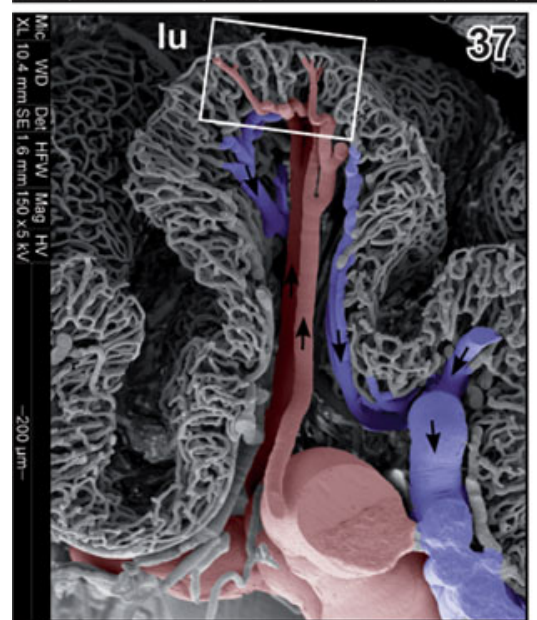

Figs. 36-38 Fig. 36 Microvascular pattern of gastric mucosal folds $(m f)$ in adult Xenopus. Detail from a transverse section. Note strongly undulating arteries $(a)$ with ascending arterioles $(a a)$. $l u$ Lumen, $m l$ muscular layer (muscularis), sm submucosa. Fig. 37 Microvascular pattern of a single mucosal fold. Detail from Fig. 33 (boxed area). Note two ascending arterioles (colored red) and two descending venules (colored blue) supplying/draining the mucosal capillary bed.

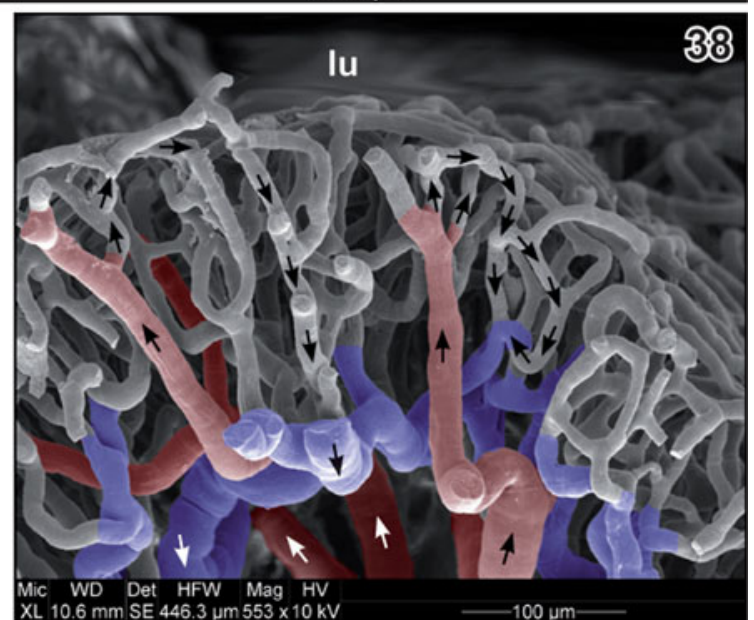

Arrows indicate direction of blood flow. lu Lumen. Fig. 38 Microvascular pattern of the mucosa of the stomach in adult Xenopus. Detail from Fig. 37 (boxed area). Note that ascending arterioles (colored red) capillarize close below the mucosal epithelium. Mucosal capillaries descend centrifugally and drain into venules (colored blue) located at the base of the mucosa. Arrows indicate direction of blood flow. $l u$ Lumen 
predominantly circumferentially to obliquely (Figs. 29-31). The muscular layer was well vascularized (Figs. 30, 31, 32) via only a few arterioles, which arose from submucosal arteries and run centrifugally (Fig. 31). Muscular capillaries ran parallel to the muscle cells and formed a rectangular network whereby draining venules by far outnumbered supplying arterioles (Fig. 31). Branches of submucosal arteries ascended towards the base of the lamina propria (Figs. 32-39) and gave off arterioles which either ascended straight (Figs. 36-40) or slightly obliquely (Fig. 41) towards the base of the gastric epithelium. There arterioles branched into wide capillaries which descended and formed the capillary bed embracing tubular gastric glands (Fig. 39). Mucosal venules descended further and formed at the base of the mucosa larger venules (Figs. 37-40) which merged, descended further and finally formed the gastric veins (Figs. 30, 34, 35).
When vascular casts were studied from the luminal side it became evident that the wide subepithelial capillaries formed ring-like patterns whereby some wider capillaries formed larger polygonal meshes enclosing several smaller meshes (Fig. 42). The wider capillaries were identified as a direct continuation of the ascending arterioles (Fig. 41).

At the transition zone to the duodenum, the gastric mucosal vascular patterns changed within a short distance into the intestinal mucosal pattern with undulating intestinal folds of varying height and orientation (not shown).

\section{Discussion}

Scanning electron microscopy (SEM) of vascular corrosion casts (VCC) has been used for over four decades (Murakami 1971) to study the microvasculature of tissues and
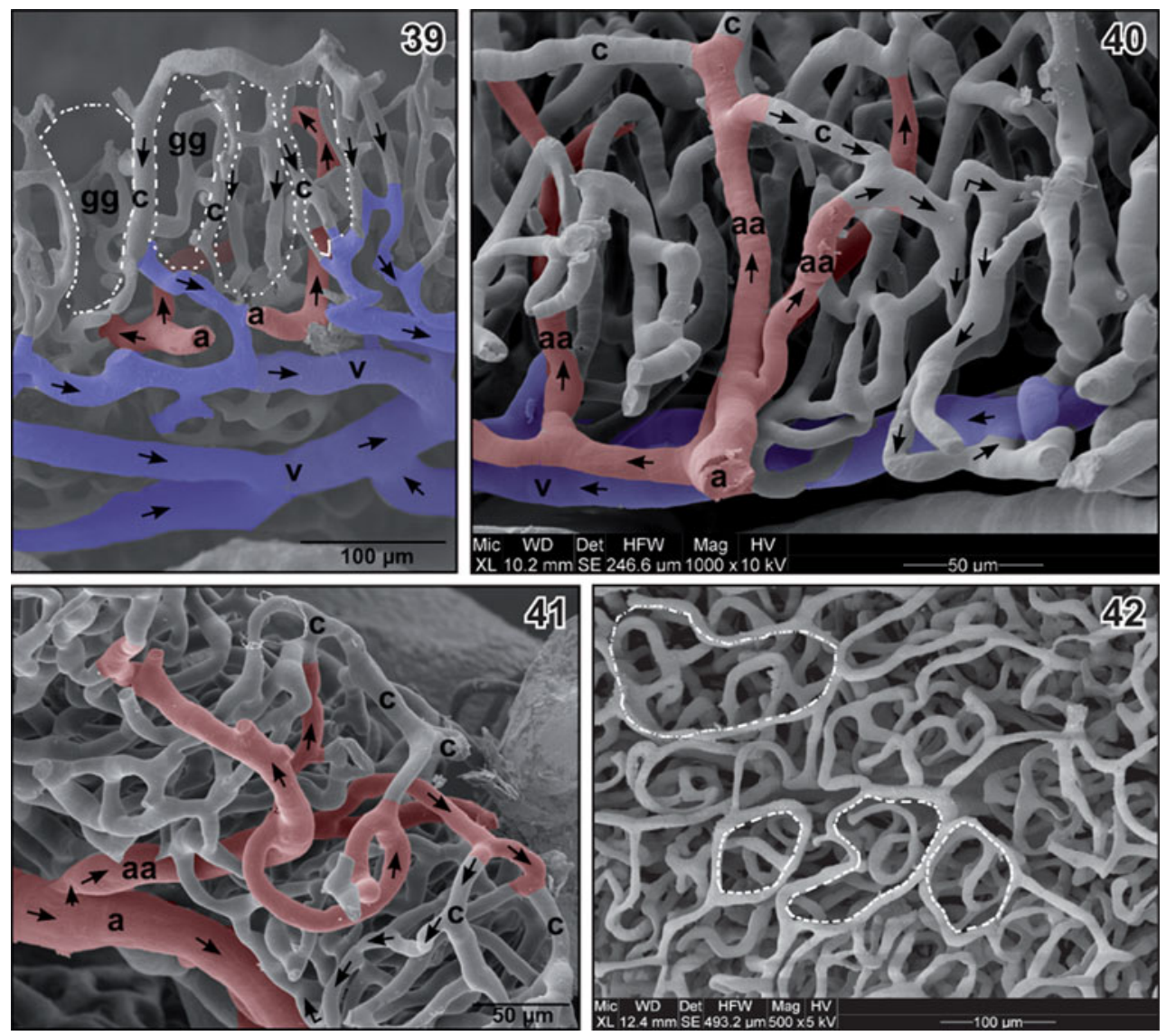

Figs. 39-42 Fig. 39 Microvascular patterns of the gastric mucosa in adult Xenopus. Detail from a transverse sectioned stomach. Oblique serosal view. Note small submucosal arteries $(a)$, ascending arterioles (colored red), descending venules and submucosal small veins (v) (colored blue). Dashed lines outline gastric glands ( $g g$ ) ensheathed by descending capillaries $(c)$. Arrows indicate direction of blood flow. Fig. 40 Microvascular patterns of the gastric mucosa in adult Xenopus. The ascending arteriole $(a a)$ branches terminally into subepithelial capillaries $(c)$. Arteries $(a)$ are red, veins $(v)$ are blue.
Arrows indicate direction of blood flow. Fig. 41 Same as Figs. 39 and 40 , but arteriole ( $a a$ ) ascends slightly obliquely and continues into the uppermost subepithelial capillary meshes $(c)$. Note the narrowing (arrowhead) at the origin of the ascending arteriole (aa) from the submucosal artery (a). Arrows indicate direction of blood flow. Fig. 42 Vascular patterns of the gastric mucosa in adult Xenopus. Luminal view. Note the large capillary meshes (outlined by dashed lines) encircling smaller ones 
organs in great detail. While VCC of fully differentiated (mature) arteries and veins can be differentiated easily by their characteristic ECNI (Figs. 1, 2), undifferentiated (immature) vessels often lack these imprints and VCC can be identified either only by their branching/merging patterns or solely by tracing them to their origin from a major vessel that can be positively identified as artery or vein.

Because during cast processing all organic materials are removed by maceration and thus anatomical landmarks are lost, a second technique - in our case histomorphology - is used to identify these landmarks and to attribute cast blood vessels to the tissue layers of the stomach.

Another concern in SEM of VCC is the interpretation of cast vascular structures and their identification as artifacts or real vascular structures (for further discussion, see e.g., Lametschwandtner and Lametschwandtner 1992; Lametschwandtner and Aharinejad 1997; Aharinejad and Böck 1994). This concern is of particular importance when studying vascular morphogenesis, as in this study. As in developing organs with growing vascular networks blind ending vessels can represent either insufficiently filled vessels or vascular sprouts, the tips of cast blind ending vessels were carefully inspected in the SEM. By general consensus, cast vessels with tapering or sharp tipped endings are considered to be vascular sprouts and as such real existing structures, while vessels that end abruptly and had a smooth, rounded end are taken as insufficiently filled vessels, and vessels that ended with a sharp cut surface are considered to be vessels broken during cast processing and thus represent artifacts.

At present, few SEM studies focus on the microvascular anatomy of the stomach of vertebrates. Of particular interest are the findings of Aharinejad et al. (1994), who reported that, in the guinea pig, gastric mucosal vascular patterns extended into the duodenum and surrounded, peninsula-like, the orifice of the common bile duct. The authors hypothesized that the "gastric vascular pattern" might play a protective role for the mucosa surrounding the common bile duct orifice. This contrasts strongly with the situation in X. laevis where (1) the gastric mucosal capillary pattern changed into the intestinal capillary pattern within a short clearly demarcated transition zone, and (2) the common bile duct opened at the crest of an intestinal fold (not shown).

The present study showed clearly that, in late prometamorphosis/early metamorphic climax (stages 55-57), the blood vessels of the stomach of Хеnориs were still immature. That is, capillaries were wide and the vasculature lacked a clear hierarchical structure. For a comparison of immature and mature vessels, particularly of capillaries, see Figs. 24 and 31. Many signs of IMG stressed the importance of non-sprouting angiogenesis in growth and differentiation of gastric vessels. In late climax (stage 64), most blood vessels were mature, and cast arteries and veins could be differentiated, by both their characteristic ECNI and their branching/merging patterns, and the microvasculature closely resembled that in the adult animal.

In contrast to the vascular pattern found in the gastric mucosa of the adult rat (Gannon et al. 1982; Ohtani et al. 1983), where arterioles capillarized at the most abluminal aspect of the mucosa, those in X. laevis broke up at the apical (luminal) aspect and formed a fountain-like pattern with a suggested apical to basal (i.e., centrifugal) blood flow along the gastric glands (Fig. 43). A functional role of these vessels as a vascular route for an ultrashort communication between the three cell types (mucous producing neck cells closest to the lumen, $\mathrm{HCl}$-producing parietal cells in the middle position, and pepsinogen-producing chief cells most distally) of the gastric glands as proposed for the vessels of the mammalian stomach by Gannon and Perry (2011) is thus very unlikely in Xenopus.

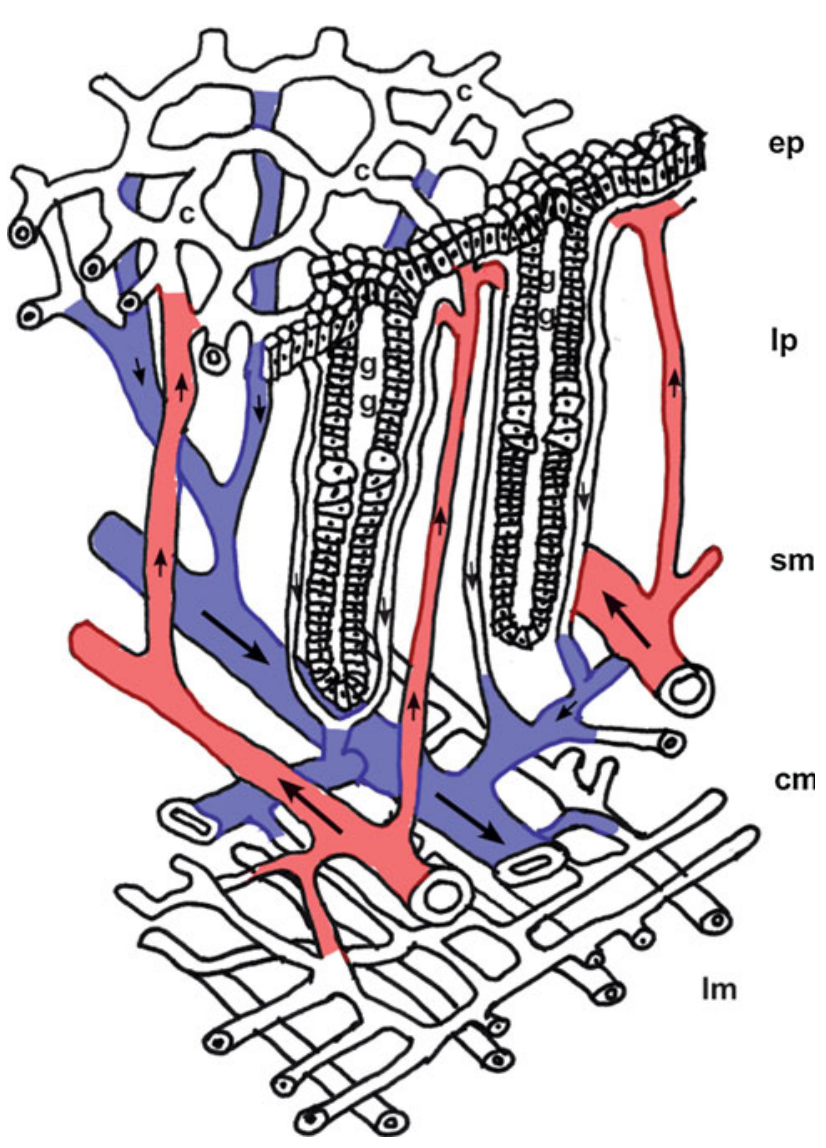

Fig. 43 Scheme depicting the blood supply of the gastric mucosa in adult Xenopus laevis. Note the ascending (centripetal) arterioles that capillarize into subepithelial capillaries $(c)$, and descending (centrifugal) venules that drain into submucosal veins. $\mathrm{cm}$ Vessels of the inner circular muscle layer, ep columnar gastric epithelium, $g g$ gastric glands, $l m$ vessels of the outer longitudinal muscle layer, $l p$ lamina propria, sm submucosa. Arrows indicate direction of blood flow 
Acknowledgments This work was supported by a grant from the Fonds zur Förderung der Wissenschaftlichen Forschung (Projekt B-19050-B17) and the Stiftungs- und Förderungsgesellschaft der Paris-Lodron-Universität Salzburg. The authors thank Dr. W.D. Krautgartner for providing excellent working conditions in the SEM facility, and Ms. Synnöve Tholo for assistance in color coding of blood vessels.

\section{Conflict of interest None.}

Open Access This article is distributed under the terms of the Creative Commons Attribution Noncommercial License which permits any noncommercial use, distribution, and reproduction in any medium, provided the original author(s) and source are credited.

\section{References}

Adam H, Czihak G (1964) Arbeitsmethoden der makroskopischen und mikroskopischen Anatomie. Fischer, Stuttgart

Aharinejad SH, Lametschwandtner A (1992) Scanning electron microscopy of vascular corrosion casts. Springer, Vienna

Aharinejad SH, Lametschwandtner A, Böck P, Firbas W (1994) Microangioarchitecture of the guinea pig common bile duct and duodenal papilla: a scanning electron and light microscopic study. Anat Rec 239:280-286

Aharinejad SH, Böck F (1994) Different forms of corrosion casts. Scanning Microsc 8:403-414

Böck P (ed) (1989) Romeis-Mikroskopische Technik. Urban \& Schwarzenberg, München

Burri P, Hlushchuk R, Djonov V (2004) Intussusceptive amgiogenesis: its emergence, its characteristics, and its significance. Dev Dyn 231:474-488

Djonov V, Makanya AN (2004) New insights into intussusceptive. Angiogenesis. In: Clauss M, Breier G (eds) Mechanisms of angiogenesis. Birkhäuser, Basel, pp 17-33

Gannon BJ, Perry MA (2011) Histoanatomy and ultrastructure of vasculature of alimentary tract. In: Comprehensive Physiology, Supplement 16: Handbook of Physiology, The Gastrointestinal System, Motility and Circulation. Wiley-Blackwell/American Physiological Society, New York, pp 1301-1334. First published in print 1989. doi: 10.1002/cphy.cp060136

Gannon BL, Browning J, O‘Brian P (1982) The microvascular architecture of the glandular mucosa of rat stomach. J Anat 135:667-683

Lambertini G (1929) Il manicotto glandulare di Rana esculenta nei suoi aspetti strutturali e nelle sue evoluzioni metamorfiche durante lo sviluppo. Ric Morfol Roma 9:71-88

Lametschwandtner A, Simonsberger P, Adam H (1980) On the prevention of specimen charging in scanning electron microscopy of vascular corrosion casts by attaching conductive bridges. Mikroskopie 36:270-273

Lametschwandtner A, Lametschwandtner U, Weiger T (1990) Scanning electron microscopy of vascular corrosion casts- technique and applications: updated review. Scanning Microsc 4:889-941

Lametschwandtner A, Lametschwandtner U (1992) Historical review and technical survey of vascular casting and scanning electron microscopy. In: Motta PM, Murakami T, Fujita H (eds) Scanning electron microscopy of vascular casts: methods and applications. Kluwer, Boston, pp 1-11

Lametschwandtner A, Aharinejad SH (1997) Scanning electron microscopy/corrosion casting technique in biological and medical research. State of the art and perspectives. In: Motta PM (ed) Recent advances in microscopy of cells, tissues and organs. Antoni Delfino Editore, Rome, pp 51-58

Levine AJ, Munoz-Sanjuan I, Bell E, North AJ, Brivanlou AH (2003) Fluorescent labeling of endothelial cells allows in vivo, continuous characterization of the vascular development of Xenopus laevis. Dev Biol 254:50-67

Makanya AN, Hlushchuk R, Djonov VG (2009) Intussusceptive angiogenesis and its role in vascular morphogenesis, patterning, and remodeling. Angiogenesis 12:113-123

Millard N (1941) The vascular anatomy of Xenopus laevis (Daudin). Trans R Soc S Afr 28:387-439

Millard N (1942) Abnormalities and variations in the vascular system of Xenopus laevis (Daudin). Trans R Soc S Afr 29:9-28

Millard N (1945) The development of the arterial system of Xenopus laevis (Daudin) including experiments on the destruction of the larval aortic arches. Trans R Soc S Afr 30:217-234

Millard N (1949) The development of the venous system of Xenopus laevis (Daudin). Trans R Soc S Afr 32:55-97

Motta PM, Murakami T, Fujita H (eds) (1992) Scanning electron microscopy of vascular casts: methods and applications. Kluwer, Boston

Murakami T (1971) Application of the scanning electron microscope to the study of the fine distribution of the blood vessels. Arch Histol Jpn 32:445-454

Nieuwkopp PD, Faber J (1967) A normal table of Xenopus development (Daudin). North Holland, Amsterdam

Ohtani O, Kikuta A, Ohtsuka A, Taguchi T, Murakami T (1983) Microvasculature as studied by the microvascular corrosion casting/scanning electron microscope method. I. Endocrine and digestive system. Arch Histol Jpn 46:1-42

Patan S, Alvarez MJ, Schittny JC, Burri PH (1992) Intussusceptive microvascular growth: a common alternative to capillary sprouting. Arch Histol Jpn 55:65-75

Ueck M (1967) Der Manicotto glandulare ("Drüsensenmagen") der Anurenlarve in Bau, Funktion und Beziehung zur Gesamtlänge des Darmes. Eine mikroskopisch-anatomische, histochemische und elektronenoptische Studie an der omnivoren und mikrophagen Larve von Xenopus laevis und der carnivoren und makrophagen Larve von Hymenochirus boettgeri (Anura, Pipidae). Z Wiss Zool 176:173-270

Weisz P (1945a) The development and morphology of the larva of the South African Clawed Toad, Xenopus laevis. I. The third form tadpole. J Morphol 77:163-192

Weisz P (1945b) The development and morphology of the larva of the South African Clawed Toad, Xenopus laevis. II. The hatching and the first and second-form tadpole. J Morphol 77:193-217 